\title{
Associação da capacidade funcional com nível de exercício físico de idosos
}

\author{
Association of functional capacity with physical exercise of elderly
}

\section{Thiago Neves ${ }^{1}$ \\ Laudyceia Mioto Silva ${ }^{2}$ \\ Anna Carolina Daltro Pereira ${ }^{3}$}

Juari José Regis ${ }^{4}$

Endereço de correspondência: Thiago Neves, Av. Bandeirantes, 3900 - FMRP Anexo A

Monte Alegre, Ribeirão Preto - SP. CEP 14049-900 (65) 99918-9709

thiago.alimt@gmail.com

\begin{abstract}
Resumo
Introdução: o envelhecimento aliado ao sedentarismo tem como consequência, a diminuição do desempenho motor na realização das atividades da vida diária (AVD). Objetivo: Associar a capacidade funcional com o nível de exercício físico de idosos, participantes de um grupo de convivência da cidade de Diamantino, Mato Grosso, Brasil.

Métodos: estudo transversal com 94 idosos ( $\geq 60$ anos) participantes de um grupo de convivência da cidade de Diamantino, Mato Grosso, Brasil. Utilizou-se um questionário para coletar informações sobre perfil sociodemográfico, comportamentais, condições de saúde física, nível de exercício físico (EF), além dos instrumentos que que mediram as atividades básicas (ABVD) e instrumentais (AIVD) da vida diária. O teste Qui-quadrado foi utilizado para analisar a associação dos níveis de EF com as ABVD e AIVD.

Resultados: a média de idade foi de 69,16 $\pm 7,59$ anos, com o IMC elevado, classificando-os com excesso de peso. Houve maior predominância de mulheres, viúvas, mulatas/caboclas/pardas, baixa escolaridade e renda, com problemas cardiovasculares evidentes. Encontrou-se idosos com níveis insuficientes de EF $(20,2 \%)$. O nível satisfatório de exercícios físicos foi associado com as AIVD, mas não com as ABVD. Conclusão: houve associação dos níveis EF com as AIVD, sendoe percentual de idosos com níveis insuficiente de EF com dependências parciais nas AIVD. Ao avaliar a capacidade funcional e o estilo de vida dos idosos, pode-se buscar e planejar estratégias que retardem o aparecimento das incapacidades, e viabilizem a reabilitação quando estas forem detectadas. Além de estimular a prática de EF que atinja os níveis aceitáveis e consequentemente influencie na qualidade de vida dos idosos.
\end{abstract}

Descritores: Expectativa de vida ativa. Exercício. Idoso.

\section{Abstract}

Introduction: Aging coupled with physical inactivity results in decreased motor performance in the activities of daily living (ADL).

Objective: To associate the functional capacity with the level of physical exercise of the elderly, participants of a senior group of the city of Diamantino, Mato Grosso, Brazil.

Methods: Cross-sectional study with 94 elderly ( $\geq 60$ years old) participants of a senior group from the city of Diamantino, Mato Grosso, Brazil. A questionnaire was used to collect information on sociodemographic profile, behavioral, physical health conditions, physical exercise (PE) level, and instruments that measured the basic activities (BADL) and instrumental (IADL) of daily living. The chisquare test was used to analyze the association of PE levels with BADL and IADL.

Results: The mean age was $69.16 \pm 7.59$ years, with a high BMI, which was classified as overweight There was a greater predominance of women, widows, mulattos/caboclas/browns, low schooling and income, with evident cardiovascular problems. Even though we are part of an elderly group, we found elderly people with insufficient levels of PE (20.2\%). The satisfactory level of PE was associated with the IADL, but not with BADL.

Conclusion: There was an association of PE levels with IADLs and a large percentage of elderly people with insufficient levels of PE with partial dependencies in IADLs. To evaluate the functional capacity and lifestyle of the elderly, strategies can be found that delay the onset of disability, and enable rehabilitation when they are detected. In addition to stimulating the practice of PE that reaches acceptable levels and consequently influences the quality of life of the elderly.

Keywords: Active life expectancy. Exercise. Elderly.

\section{Cite como}

Neves T, Silva LM, Pereira ACD, Régis JJ Associação da capacidade funcional com nível de exercício físico de idosos Conscientiae Saúde 2019 jul./set.; 18(3):339-351. https://doi.org/10.5585/ConsSaude.v18n3 13755. 


\section{Introdução}

Nos dias atuais, com a diminuição das taxas de fertilidade e o aumento da expectativa de vida, os idosos podem viver aproximadamente vinte anos além dos sessenta ${ }^{1}$. Estima que até o ano de 2050 a população mundial de idosos com mais de sessenta anos vai passar dos atuais 841 milhões para 2 bilhões, evidenciando uma preocupação constante com o atendimento destes pela saúde pública, pois o fato de viver mais não implica em um envelhecimento saudável ${ }^{2}$.

O envelhecimento proporciona a ocorrência de várias mudanças físicas e mentais. Tratando especialmente nas mudanças físicas, tem-se o envelhecimento dos órgãos, percebe-se a diminuição das capacidades físicas como velocidade, força, resistência, agilidade, equilíbrio, diminuição da acuidade visual, olfato, audição e paladar ${ }^{3}$. Evidencia-se que a capacidade funcional se refere a fatores explicativos como à inclusão de comportamentos saudáveis, ou não, relacionados ao estilo de vida ${ }^{4,5}$. Podendo ser mantidas através de hábitos saudáveis, como ter uma alimentação saudável, não ingerir bebidas alcoólicas, não fumar e praticar exercícios físicos, sendo estes indicadores de grandes benefícios para o envelhecimento saudável ${ }^{6}$.

Para mensuração da dependência física, especialmente em pessoas idosas, há uma variedade de instrumentos, porém, ainda não existe um padrão ouro de avaliação ${ }^{7}$. Mesmo assim, alguns instrumentos são considerados válidos e confiáveis, sendo as mais utilizadas as ABVD (Escala de Katz) $)^{8}$, as AIVDs (Escala de Lawton e Brody) ${ }^{9}$ e a mobilidade ${ }^{10}$. Além disso, o envelhecimento aliado ao sedentarismo tem como consequência, a diminuição do desempenho motor na realização das atividades da vida diária (AVD), mas não necessariamente, dependentes de outros ${ }^{11}$.

Estudos realizados de pesquisas populacionais transversais, desenvolvidas em regiões diferenciadas no Brasil apresentaram que idosos da região urbana possuem maior dependência grave que aqueles da região rural $^{12}$. Além de existirem diferentes métodos utilizados para avaliar a capacidade funcional em idosos brasileiros, que dificulta a comparação de resultados entre os $\operatorname{artigos}^{13}$. Demonstrando em seus resultados a heterogeneidade do processo de envelhecimento, levando a se recomendar o planejamento de políticas e ações de saúde a esse grupo etário em diversas regiões do pais $^{12,13}$.

Devido à escassez de estudos com idosos e sua capacidade funcional na região do interior de Mato Grosso, por terem aspectos culturais e alimentares diferentes, percebemos a necessidade de realizar a pesquisa com intuito de contribuir para diagnósticos práticos e precisos, para possíveis construções de novas estratégias e caracterizando o perfil físico e 
funcional desses idosos. Como objetivo, busca-se verificar a associação da capacidade funcional com os níveis de exercício físico de dos idosos frequentadores de um grupo voltados para terceira idade da cidade de Diamantino - MT.

\section{Métodos}

Esta investigação foi realizada a partir dos dados contidos no banco eletrônico do estudo PRIMO (Projeto Idoso com Muito Orgulho), relativos à cidade de Diamantino, Mato Grosso, Brasil, no contexto de um estudo multicêntrico, populacional, de coorte, que teve como finalidade investigar a influência de programas de exercícios físicos orientados e estruturados em indivíduos idosos ( $\geq 60$ anos) da comunidade participantes de centros de convivências do município de Diamantino e adjacentes/Mato Grosso, cujo projeto foi submetido ao Comitê de Ética em Pesquisas da Universidade Estadual de Mato Grosso (UNEMAT) e aprovado mediante o parecer $n^{\circ} .2 .451 .454 / 2017$.

O estudo é caracterizado por ser transversal de caráter quantitativo, descritivoexploratório. A população foi constituída por idosos participantes de um grupo de convivência, voltado para terceira idade no município de Diamantino. No grupo conta com 419 idosos cadastrados e 130 participantes assíduos.

Foram incluídos no estudo, os idosos que estivessem com frequência constante e que estivessem nos dias programados para coleta de dados e ter idade igual ou superior a sessenta anos, além de dar o seu consentimento para participar do estudo assinando termo de consentimento livre e esclarecido (TCLE). Como critérios de exclusão, definiram-se os indivíduos que não tinham capacidade de comunicação verbal ou escrita e de compreensão do TCLE e dos questionários. Após os critérios de seleção, o estudo teve a participação 94 idosos, sendo composto na maioria por mulheres $(91,5 \%)$.

Para coleta dos dados utilizou-se o questionário composto por questões sócio demográficas, anamnese clínica, nível de atividade física e capacidade funcional. A quantificação do nível de exercício físico (EF), foi empregado o International Physical Activity Questionnaire (IPAQ), em sua versão curta, validado no Brasil, que objetiva estimar o nível da prática habitual de exercícios físicos, sendo uma alternativa para diversos estudos, pois possibilita maior praticidade, rapidez e permite levantamentos de grandes grupos populacionais, além de possuir coeficientes de validação e reprodutividade similares aos de

outros instrumentos ${ }^{11}$, possuindo, em sua composição, questões sobre duração, frequiência, 
intensidade e tipo de atividade, permitindo estimar o gasto calórico total, o que acaba facilitando também a classificação das atividades como leves, moderadas e vigorosas, conforme instrução própria da ferramenta, sendo considerados:

a) Sedentários: indivíduos com menos de 10 minutos/semana de EF contínua; b) Insuficientemente ativo A: indivíduos com mais de 10 minutos/semana de EF contínua - 5 dias/semana ou 150 minutos/semana; c) Insuficientemente ativo B: indivíduos que não atinge nenhum dos critérios para ser classificado como Insuficientemente ativo A; d) Ativo AF vigorosa $\geq 3$ dias/semana e $\geq 20$ minutos/sessão - Moderada ou caminhada $\geq 5$ dias/semana e $\geq 30$ minutos/sessão - Qualquer atividade somada $\geq 5$ dias/ semana e $\geq 150$ minutos/ semana; e) Muito Ativo EF vigorosa $\geq 5$ dias/semana e $\geq 30$ minutos/ sessão - EF vigorosa $\geq 3$ dias/ semana e $\geq 20$ minutos/sessão + moderada e ou caminhada $\geq 5$ dias na semana e $\geq 30$ minutos.

A capacidade funcional dos idosos foi avaliada pela escala de $\mathrm{Katz}^{8}$ que avalia as atividades básicas da vida diária (ABVD) e Lawton e Brody ${ }^{9}$ que avalia as atividades instrumentais da vida diária (AIVD). Os entrevistados foram perguntados se eles tinham dificuldades em realizar as ABVD (transferência, ir ao banheiro, tomar banho, continência urinária, vestir-se e alimentação) e as AIVD (usar um telefone, usar o transporte, fazer compras, preparar refeições, realizar tarefas domésticas leves, fazer tarefas domésticas pesadas, tomar medicamentos e administrar o dinheiro).

Para análise dos resultados e uma melhor interpretação, os dados obtidos pelo IPAQ foram subdivididos em dois grupos: Níveis Insuficientes de Exercício Físico (NIEF) e Níveis Suficientes de Exercício Físico (NSEF) O primeiro grupo foi composto a classificação dos resultados entre sedentário, insuficientemente ativo " $\mathrm{A}$ " e insuficientemente ativo " $\mathrm{B}$ ". $\mathrm{O}$ segundo grupo incluiu o restante das classificações de "Ativo" e "Muito ativo".

Para caracterização da amostra foi feita uma análise descritiva por médias, desvio padrão, frequência absoluta e relativa (percentuais) das variáveis. Utilizou-se o teste Qui-quadrado para analisar a associação dos níveis de EF com as ABVDs e AIVDs. Em todas as análises, adotouse o nível de significância estatística de $5 \%(\mathrm{p} \leq 0,05)$. As análises e o tratamento estatístico dos dados foram realizadas com auxílio do software Statistical Package for the Social Sciences, versão 25.0 for Windows®. 


\section{Resultados}

Dos 94 idosos que participaram do estudo, a média de idade foi de 69 anos, com o IMC elevado, classificando-os com excesso de peso. Houve maior predominância de mulheres, de casados (as) e viúvos (as), mulato/caboclo/pardo (a). A maioria dos entrevistados tem baixa escolaridade e renda, com problemas cardiovasculares, tendo níveis suficientes de exercícios físicos elevados (Tabela 1).

Tabela 1 - Perfil sociodemográfico dos idosos. Diamantino/MT, 2017

\begin{tabular}{ll}
\hline Variáveis & Média \pm Desvio Padrão \\
Idade & $69,16 \pm 7,59$ \\
Índice de massa corporal $\left(\mathrm{km} / \mathrm{m}^{2}\right)$ & $27,36 \pm 4,90$ \\
\hline Gênero & Número $(\%)$ \\
\hline Masculino & $8(8,5)$ \\
Feminino & $86(91,5)$ \\
\hline Estado civil & \\
\hline Solteiro (a) & $6(6,4)$ \\
Casado (a) & $35(37,2)$ \\
Viúvo (a) & $42(44,7)$ \\
Divorciado (a) & $11(11,7)$ \\
\hline Raça & \\
\hline Branco (a) & $33(35,1)$ \\
Preto ou Negro (a) & $14(14,9)$ \\
Mulato/caboclo/pardo (a) & $45(47,9)$ \\
Indígena & $2(2,1)$ \\
\hline Escolaridade (anos) & \\
\hline 0 a 8 anos & $85(90,4)$ \\
9 a 11 anos & $4(4,3)$ \\
$\geq 12$ anos & $5(5,3)$ \\
\hline Renda (salários mínimos)* & \\
\hline < 1 & $3(3,2)$ \\
1 a 2 & $65(69,1)$ \\
2 a 5 & $25(26,6)$ \\
> 5 & $1(1,1)$ \\
\hline Problemas de Saúde & \\
\hline Cardiovasculares & $63(67,0)$ \\
Pulmonares & $4(4,3)$ \\
Osteo-articulares & $8(8,5)$ \\
Metabólico-endócrinas & $18(19,1)$ \\
\hline International Physical Activity Questionnaire (IPAQ) \\
\hline Níveis insuficientes de exercícios físicos \\
Níveis suficientes de exercícios físicos & $75(79,8)$ \\
& \\
\hline & \\
\hline
\end{tabular}

Fonte: Autoria própria. 
Em relação à funcionalidade dos idosos nas ABVD, conforme mostra a Tabela 2, em todas atividades tiveram idosos que precisavam de ajuda, principalmente para se vestir $(2,8 \%)$ e algum tipo de incontinência para as micções e/ou para as evacuações $(2,8 \%)$.

Nas AIVD, conforme mostra a Tabela 3, em todas atividades tiveram idosos que precisavam de ajuda, principalmente para fazer os trabalhos manuais domésticos $(12,8 \%)$, arrumar a casa $(8,5 \%)$, entretanto, as atividades de preparar suas próprias refeições $(3,2 \%)$ e tomar seus remédios na dose e horários corretos $(4,3 \%)$, foram as que menos precisavam de ajuda.

Tabela 2 - Perfil das Atividades básicas da vida diária dos idosos. Diamantino/MT, 2017

\begin{tabular}{lll}
\hline \multirow{2}{*}{ Atividades básicas da vida diária } & Com ajuda & Sem ajuda \\
\hline Tomar banho? & $\mathrm{N}(\%)$ & $\mathrm{N}(\%)$ \\
Se vestir? & $1(1,1)$ & $93(98,9)$ \\
Ir ao banheiro? & $2(2,1)$ & $92(97,9)$ \\
Transferência (sentar, levantar da cama ou cadeira)? & $1(1,1)$ & $93(98,9)$ \\
Continência (urinar e evacuar)? & $1(1,1)$ & $93(98,9)$ \\
Se alimentar? & $2(2,1)$ & $92(97,9)$ \\
\hline
\end{tabular}

Fonte: Autoria própria.

Já nas atividades para as quais alguns idosos eram totalmente dependentes incluíam todas elas novamente, porém com percentuais menores, sendo mais relatadas: fazer os trabalhos manuais domésticos $(5,3 \%)$, tomar seus remédios na dose e horários corretos $(5,3 \%)$, usar o telefone $(4,3 \%)$ e lavar e passar a sua roupa $(4,3 \%)$.

Tabela 3 - Perfil das Atividades Instrumentais da vida diária dos idosos. Diamantino/MT, 2017

\begin{tabular}{llll}
\hline Atividades instrumentais da vida diária & $\begin{array}{l}\text { Não } \\
\text { consegue } \\
\mathrm{N}(\%)\end{array}$ & $\begin{array}{l}\text { Com } \\
\text { ajuda } \\
\mathrm{N}(\%)\end{array}$ & $\begin{array}{l}\text { Sem } \\
\text { ajuda } \\
\mathrm{N}(\%)\end{array}$ \\
\hline Consegue usar o telefone? & $4(4,3)$ & $6(6,4)$ & $84(89,4)$ \\
Consegue ir a locais distantes, sem planejamento? & $2(2,1)$ & $7(7,4)$ & $85(90,4)$ \\
Consegue fazer compras? & $1(1,1)$ & $7(7,4)$ & $86(91,5)$ \\
Consegue preparar suas próprias refeições? & $2(2,1)$ & $3(3,2)$ & $94(94,7)$ \\
Consegue arrumar a casa? & $2(2,1)$ & $8(8,5)$ & $84(89,4)$ \\
Consegue fazer os trabalhos manuais domésticos? & $5(5,3)$ & $12(12,8)$ & $77(81,9)$ \\
Consegue lavar e passar a sua roupa? & $4(4,3)$ & $6(6,4)$ & $84(89,4)$ \\
Consegue tomar seus remédios na dose e horários \\
corretos?
\end{tabular}

Fonte: Autoria própria. 
Na Figura 1, estão expressas ABVD com o IPAQ foi expressa. Não encontrou associação significativa dos níveis de exercícios físicos com as ABVD ( $p>0,05)$.

Na Figura 2, a relação da AIVD com o IPAQ. Nota-se associação significativa dos níveis de exercícios físicos com as AIVD ( $\mathrm{p}<0,05)$, um grande percentual de idosos com NIEF com dependências parciais nas AIVD.

Figura 1 - Associação dos níveis de exercício físico observado nos idosos segundo a classificação funcional nas atividades básicas da vida diária (ABVD). $\mathrm{p}=0,376$ pelo teste Qui-quadrado. IPAQ: International Physical Activity Questionnaire; NIEF: Nível Insuficiente de Exercício Físico; NSEF: Nível Suficiente de Exercício Físico

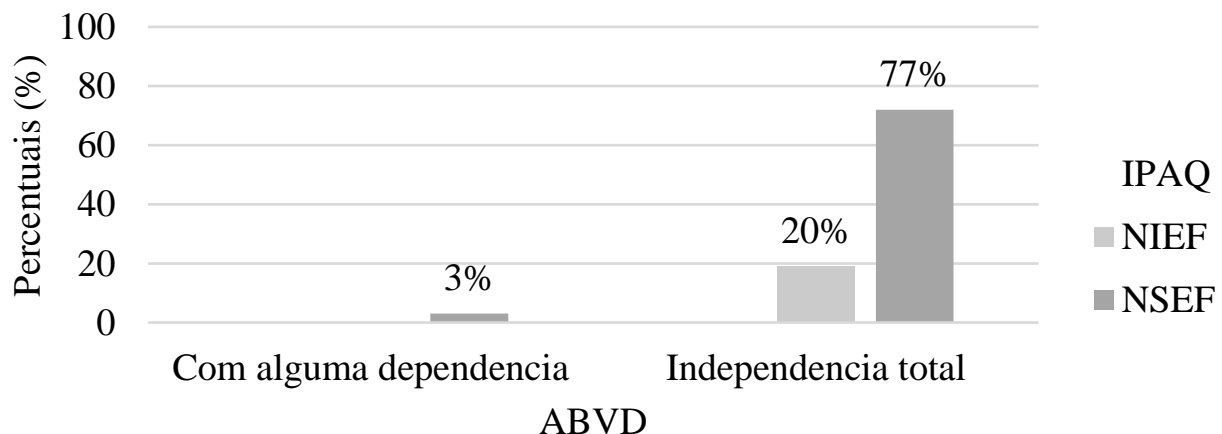

Fonte: Autoria própria.

Figura 2 - Associação dos níveis de exercício físico observado nos idosos segundo a classificação funcional nas atividades instrumentais da vida diária (AIVD). $\mathrm{p}=0,020$ pelo teste Quiquadrado. IPAQ: International Physical Activity Questionnaire; NIEF: Nível Insuficiente de Exercício Físico; NSEF: Nível Suficiente de Exercício Físico.

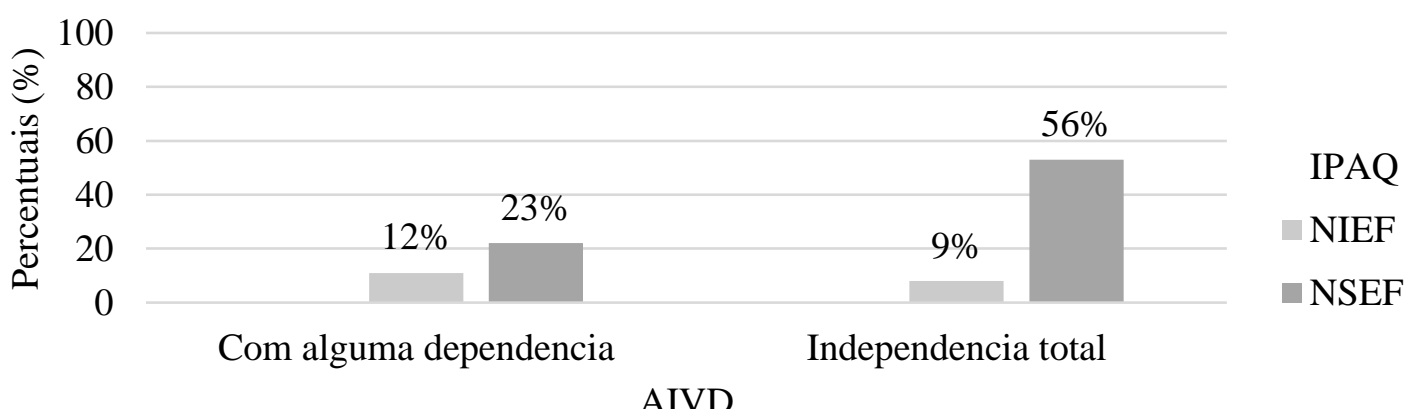

Fonte: Autoria própria 


\section{Discussão}

A média de idade dos idosos foram 69 anos, com o IMC elevado, ou seja, a maioria com excesso de peso. A maior predominância foi de mulheres, viúvas, mulatas/caboclas/pardas, baixa escolaridade e renda, com problemas cardiovasculares evidentes, além de terem NSEF elevados.

De acordo com alguns estudos, a maioria dos frequentadores de grupos de convivência são mulheres casadas ${ }^{14,15}$, com faixa etária entre 60-69 anos, bom nível de escolaridade ${ }^{16,17}$ e renda entre um e dois salários mínimos ${ }^{18}$. O presente estudo teve um bom número de casados (as), porém a maior parte eram viúvos (as), sendo semelhantes os resultados de baixa escolaridade e renda. Alguns grupos de voltados para idosos o público feminino é o mais prevalente, corroborando outros estudos ${ }^{14,19-22}$. Os resultados do presente estudo identificaram que grande parte da amostra se encontrou com excesso de peso, corroborando com outros estudos que também encontraram essa mesma situação ${ }^{23,24}$.

Mesmo fazendo parte de um grupo voltado para terceira idade, encontramos idosos com níveis insuficientes de EF (20,2\%). Uma possível explicação disso, pode estar relacionada com a prática dos idosos em atividades que não exigem esforços físicos suficientes para serem classificados como suficientemente ativos pelo IPAQ. Comparando os resultados deste estudo com outros, verifica-se que neste estudo a prevalência de idosos insuficientemente ativos é baixa. Levando em conta a prevalência de inatividade física insuficiente no estudo realizado na população da região urbana de Montes Claros, Minas Gerais foi de $57,1 \%$ nos idosos ${ }^{23}$. Este presente estudo apresenta um índice inferior aos demonstrados pelo inquérito realizado pelo VIGITEL $^{25}$. No inquérito a frequência de adultos que não praticam o volume recomendado de exercícios físicos no tempo livre foi de $69,7 \%$.

Em outros estudos do tipo transversal e de base populacional, um realizado em 42 cidades em sete estados brasileiros, com uma amostra de 4.060 adultos e 4.003 idosos; identificou-se a inatividade física em $58 \%$ dos entrevistados ${ }^{26}$ e outro estudo investigou a inatividade física e alguns fatores associados em 1.018 adultos e 1.010 idosos, em 10 municípios em Pernambuco, verificou que $68,3 \%$ dos idosos eram fisicamente inativos ${ }^{7}$.

Alguns fatores podem explicar alguns níveis de prática ou não dos exercícios físicos na população de idosos, um deles é a questão do poder aquisitivo. É importante destacar que o tipo de exercício físico realizado pelas pessoas tende a variar de acordo com o nível 
socioeconômico. No presente estudo, essa questão econômica não foi um fator limitante na realização de exercício físico, mesmo grande parte da amostra receber de 1 a 2 salários mínimos. Entretanto, isso de alguma maneira pode interferir na prática de algum tipo de exercício físico, como na compra de algum acessório (tênis, agasalho, dentre outros), ou aqueles exercícios físicos que demandasse dinheiro na sua realização, limitando assim, a adesão à essa prática ${ }^{27,28}$.

Em geral, as pessoas com maior nível socioeconômico, são mais aptas para realização de exercício físico em relação às de menor poder aquisitivo ${ }^{26}$. Todavia, outro estudo identificou que os residentes em áreas mais pobres apresentaram maior chance de caminhar ou andar de bicicleta para fazer compras ou ir trabalhar ${ }^{29}$. No atual estudo, isso pode ser um fator explicativo de alguns idosos não terem atingido os NSEF.

Em relação a capacidade funcional auto referida nas AIVD, em todas atividades os idosos precisavam de ajuda, principalmente para fazer os trabalhos manuais domésticos (12,8\%), arrumar a casa $(8,5 \%)$, diferente do estudo feito com 95 idosos da comunidade de uma área de abrangência do Programa Saúde da Família, em Goiânia que as três AIVD nas quais os idosos necessitavam mais ajuda para o manuseio de dinheiro $(73,9 \%)$, uso de meios de transporte $(72,5 \%)$ e trabalho doméstico $(40,6 \%)^{30}$.

Os resultados referentes as atividades para as quais alguns idosos eram totalmente dependentes foi fazer os trabalhos manuais domésticos (5,3\%), tomar seus remédios na dose e horários corretos $(5,3 \%)$, usar o telefone $(4,3 \%)$ e lavar e passar a sua roupa (4,3\%). Resultados correlacionados ao encontrados pelo mesmo estudo feito em Goiânia ${ }^{30}$, que observou maior dependência total em lavar qualquer peça de roupa $(30,4 \%)$, realizar trabalho doméstico $(26,1 \%)$ e utilizar o telefone $(20,3 \%)$.

Esse grau de dependência para as AIVD foi bem menor do que o identificado em dois estudos realizados no estado de Goiás ${ }^{30,31}$, na qual foi observado que mais de 55,0\% dos idosos eram dependentes em uma ou mais atividades. Em outro estudo sobre a CF em pessoas idosas, utilizando também a Escala de Lawton e de Katz, observou-se que 52,6\% das idosas entrevistadas possuíam algum nível de dependência nas AIVD e 13,2\% foram classificados como dependentes em alguma $\mathrm{ABVD}^{32}$. Nesses tipos de estudos, ambas escalas de avaliação funcional são frequentemente utilizadas, sendo, instrumentos de fáceis aplicabilidade, por serem de fácil compreensão, tanto por parte do examinador como do entrevistado ${ }^{32-34}$.

Nas ABVD, os baixos valores encontrados de dependência pode ser explicado pela natureza das tarefas executadas na avaliação da escala, pois correspondem às funções mais 
básicas no que diz respeito à sobrevivência do indivíduo e detecta comprometimentos em condições de declínios funcionais avançados, de elevados níveis de deficiência, geralmente em idosos frágeis ou institucionalizados ${ }^{35}$. Já às AIVD, por se tratar de tarefas mais complexas, que estendem os aspectos abordados pela escala das ABVD, como controlar finanças, utilizar meios de transporte e usar o telefone, os resultados de dependência foi superior à anterior, o que pode também explicar a associação da mesma com o NIEF.

Apesar do presente estudo não ter encontrado associação significativas entre o IPAQ e a Escala de Katz, outros mostraram que idosos ativos apresentam bons níveis de autonomia para o desempenho de suas atividades cotidianas, quando comparados aos idosos insuficientemente $\operatorname{ativos}^{36,37}$, utilizando os mesmo instrumentos para avaliação das ABVD e AIVD. Destaca-se que essa amostra correlacionou com estudo recente realizado com 115 idosos ( $\geq 65$ anos), que utilizaram os serviços de apoio domiciliário e centro de dia no Concelho de Coimbra, Portugal, onde também observaram uma relação significativa entre as AIVD e os níveis de atividade física, em que maior independência foi encontrada nos idosos que praticavam exercício físico ${ }^{38}$.

Utilizando o IPAQ outros autores verificaram que $64,6 \%$ da população foi classificada como muito ativa ou ativa, sendo fortemente relacionada com a independência funcional ${ }^{34}$. Outro estudo também, investigou a associação entre a atividade física e o risco de declínio funcional em uma coorte de idosos nos Estados Unidos, encontraram um resultado no qual a atividade física regular esteve associada a um menor risco de declínio funcional ${ }^{39}$. Em outro estudo realizado nos Estados Unidos, também encontrou resultados semelhantes ${ }^{39}$. Portanto, as perdas ocorrem de atividades instrumentais de vida diária para atividades básicas de vida diária, devido às AIVD exigirem maior integridade física e cognitiva comparada às $\mathrm{ABVD}^{40}$.

\section{Conclusão}

Idosos que praticavam algum tipo de exercício mantiveram uma capacidade funcional satisfatória para desempenhar as atividades relatadas. Não encontrou associação dos níveis de EF com as ABVD, o que é explicado pelo fato de a grande maioria não apresentar dificuldades nessas atividades mais comuns no seu dia-a-dia.

Os achados reforçam a importância de avaliar com instrumentos acessíveis e confiáveis a capacidade funcional e o estilo de vida dos idosos de regiões que se diversificam da grandes metrópoles e centros, podendo com isso, transformar e integrar esse tipo de avaliação e analise na rotina desses grupos de convivência. Ainda, contribuindo no planejamento de estratégias e 
intervenções adequadas, que visem ações que retardem o aparecimento das incapacidades, e viabilizar a reabilitação quando estas forem detectadas, além de estimular a pratica de exercícios físicos para que atinja os níveis aceitáveis e consequentemente influencie na qualidade de vida dos idosos.

\section{Referências}

1. World Health Organization - WHO. World report on ageing and health. World Health Organization; 2015.

2. Qadir U. UN Development Programme (UNDP). Human Development Report 2015-Work for Human Development. Pak Dev Rev. 2015;54(3):277-8.

3. Castro AA De. Qualidade de vida e capacidade funcional em idosos adeptos a educação física gerontológica. :39-55.

4. Iezzoni LI. Using administrative data to study persons with disabilities. Milbank Q. 2002 Jan;80(2):347-79.

5. Rosa TE da C, Benício MHD, Latorre M do RD de O, Ramos LR. Fatores determinantes da capacidade funcional entre idosos. Rev Saude Publica. 2003 Feb;37(1):40-8.

6. Abla O, Palmert MR. Reversal of LCH-related diabetes insipidus and reappearance of posterior pituitary bright spot with low-dose chemotherapy. Pediatr Blood Cancer [Internet]. 2012/01/10. Available from:

http://www.ncbi.nlm.nih.gov/entrez/query.fcgi? $\mathrm{cmd}=$ Retrieve \&db=PubMed\&dopt=Citation\& list_uids=22223352

7. Alves LC, Leite I da C, Machado CJ. Conceituando e mensurando a incapacidade funcional da população idosa: uma revisão de literatura. Cien Saude Colet. 2008 Aug;13(4):1199-207.

8. Katz S. Studies of Illness in the Aged. JAMA. 1963 Sep 21;185(12):914.

9. Lawton MP, Brody EM. Assessment of older people: self-maintaining and instrumental activities of daily living. Gerontologist. 1969;9(3):179-86

10. Crimmins EM, Hayward MD, Saito Y. Changing Mortality and Morbidity Rates and the Health Status and Life Expectancy of the Older Population. Demography. 1994 Feb;31(1):159.

11. Andreotti RA, Okuma SS. Validating a test battery of activities of daily living for physically independent elderly. Rev Paul Educ Física [Internet]. 1999 Jun 20;13(1):46. Available from: https://www.revistas.usp.br/rpef/article/view/137759

12. Winckler M. Idosos no meio rural: $\mathrm{u} \mathrm{m}$ a r e v i s ã o i n t e g r at i va. 
13. Cristina A, Campos V, Helena M, Almeida M De. Prevalência de incapacidade funcional por gênero em idosos brasileiros : uma revisão sistemática com metanálise. 2013;545-59.

14. Marinho da Silveira M, Silva Tavares GM, Zuppa C, Wetters Portuguez M, Gomes da Silva Filho I, Attilio De Carli G, et al. Análise da qualidade de vida de idosos frequentadores de oficinas de informática. ConScientiae Saúde. 2013;12(4).

15. Silva WF, Almeida AR, Santos DT, Silveira MB. Os benefícios da atividade física para qualidade de vida dos idosos em um clube da terceira idade na cidade de Porterinha, MG. Rev Dig Buenos Aires. 2010;15:149.

16. da Silveira MM, Pasqualotti A, Colussi EL. Envelhecimento e usuários de informática: repercussões de um programa ergonômico. Estud Interdiscip sobre o Envelhec. 2012;19(1).

17. Lopes dos Santos R, Virtuoso Júnior JS. Confiabilidade da versão brasileira da escala de atividades instrumentais da vida diária. Rev Bras em Promoção da Saúde. 2008;21(4).

18. Leite MT, Winck MT, Hildebrandt LM, Kirchner RM, Silva LAA da. Qualidade de vida e nível cognitivo de pessoas idosas participantes de grupos de convivência. Rev bras geriatr gerontol. 2012;15(3):481-92.

19. Vieira MC, Santarosa LMC. O uso do computador e da Internet e a participação em cursos de informática por idosos: meios digitais, finalidades sociais. In: Brazilian Symposium on Computers in Education (Simpósio Brasileiro de Informática na Educação-SBIE). 2009.

20. Doll J, Buaes CS. Aprendizagem em cursos de inclusão digital para pessoas adultas e idosas. Rev Bras Ciências do Envelhec Hum. 2010;6(3).

21. Bez MR, Pasqualotti PR, Passerino LM. Inclusão digital da terceira idade no centro Universitário Feevale. In: Brazilian Symposium on Computers in Education (Simpósio Brasileiro de Informática na Educação-SBIE). 2006. p. 61-70.

22. Areosa SC, Bevilacqua P, Werner J. Representações sociais do idoso que participa de grupos para terceira idade no município de Santa Cruz do Sul. Estud Interdiscip sobre o Envelhec. 2003;5.

23. Vagetti GC, de Oliveira V, Pereira Silva M, Pacífico AB, Rocha Alves Costa T, de Campos W. Associação do índice de massa corporal com a aptidão funcional de idosas participantes de um programa de atividade física. Rev Bras Geriatr e Gerontol. 2017;20(2).

24. Leite-cavalcanti C, Rios-asciutti LS. Prevalência de doenças crônicas e estado nutricional em um grupo de idosos brasileiros. 2009;11(6):865-77.

25. Brasil. Ministério da Saúde. Secretaria de Vigilância em Saúde. Secretaria de Gestão Estratégica e Participativa. Vigitel Brasil 2009: vigilância de fatores de risco e proteção para doenças crônicas por inquérito telefônico. MS Brasília; 2009.

26. Siqueira FV, Facchini LA, Piccini RX, Tomasi E, Thumé E, Silveira DS, et al. Atividade 
física em adultos e idosos residentes em áreas de abrangência de unidades básicas de saúde de municípios das regiões Sul e Nordeste do Brasil. Cad Saude Publica [Internet]. 2008 Jan;24(1):39-54. Available from:

http://www.scielo.br/scielo.php?script=sci_arttext\&pid=S0102-

$311 \mathrm{X} 2008000100005 \& \operatorname{lng}=\mathrm{pt} \& \operatorname{lng}=\mathrm{pt}$

27. Cassou AC, Fermino RC, Santos MS, Rodriguez-Añez CR, Reis RS. Barreiras para a atividade física em idosos: uma análise por grupos focais. Rev da Educ Física/UEM

[Internet]. 2008 Dec 15;19(3):353-60. Available from:

http://periodicos.uem.br/ojs/index.php/RevEducFis/article/view/3675

28. Quadros Junior AC de, Lamonato ACC, Gobbi S. Nível de escolaridade não influencia nível de atividade física em idosos. Mot Rev Educ Física. 2011;202-8.

29. van Lenthe FJ, Brug J, Mackenbach JP. Neighbourhood inequalities in physical inactivity: the role of neighbourhood attractiveness, proximity to local facilities and safety in the Netherlands. Soc Sci Med. 2005;60(4):763-75.

30. Costa EC, Kyosen Nakatani AY, Bachion MM. Capacidade de idosos da comunidade para desenvolver Atividades de Vida Diária e Atividades Instrumentais de Vida Diária. Acta Paul Enferm. 2006;19(1).

31. Nakatani AYK, Costa EFDA, Teles SA, Silva LB, Rêgo MAB, Silva e Souza AC, et al. Perfil sócio-demográfico e avaliação funcional de idosos atendidos por uma equipe de saúde da família na periferia de Goiânia, Goiás. Rev Soc Bras Clín Méd. 2003;1(5):131-6.

32. Maciel ÁCC, Guerra RO. Influência dos fatores biopsicossociais sobre a capacidade funcional de idosos residentes no nordestes do Brasil. Rev bras epidemiol. 2007;10(2):178-89.

33. Cordeiro J, Del Castillo BL, de Freitas CS, Gonçalves MP. Efeitos da atividade física na memória declarativa, capacidade funcional e qualidade de vida em idosos. Rev Bras Geriatr e Gerontol. 2014;17(3):541-52.

34. Boggio E da SB, Santos FC, Souza CM, Silva MF, Rosa PV, Rosa LHT. Análise dos fatores que interferem na capacidade funcional de idosos residentes em uma comunidade de Porto Alegre. Estud Interdiscip sobre o Envelhec. 2015;20(1

35. Thomas AG, Dennis A, Bandettini PA, Johansen-Berg H. The effects of aerobic activity on brain structure. Front Psychol. 2012;3.

36. Del Duca GF, Hallal PC, Nahas MV, da Silva MC, da Silva KS. Aspectos comportamentais e de saúde associados à incapacidade funcional em idosos: estudo de base populacional-DOI: 10.4025/reveducfis. v20i4. 7265. J Phys Educ. 2009;20(4):577-85.

37. Borges MRD, Moreira ÂK. Influências da prática de atividades físicas na terceira idade: estudo comparativo dos níveis de autonomia para o desempenho nas AVDs e AIVDs entre idosos ativos fisicamente e idosos sedentários. Motriz Rev Educ Física UNESP. 2009;15(3):562-73. 
38. Oliveira A, Nossa P, Mota-pinto A. Assessing Functional Capacity and Factors Determining Functional Decline in the Elderly: A Cross-Sectional Study Avaliação da Capacidade Funcional e Fatores Determinantes do Declínio Funcional em Idosos : Um Estudo Transversal. 2019

39.Boyle PA, Buchman AS, Wilson RS, Bienias JL, Bennett DA. Physical Activity Is Associated with Incident Disability in Community-Based Older Persons. J Am Geriatr Soc. 2007;55(2):195-201.

40. Rossi Barbosa B, Marques de Almeida J, Rossi Barbosa M, Rossi-Barbosa LAR. Avaliação da capacidade funcional dos idosos e fatores associados à incapacidade. Cien Saude Colet. 2014;19(8). 